\title{
miR-124, miR-137 and miR-340 regulate colorectal cancer growth via inhibition of the Warburg effect
}

\author{
YAN SUN $^{1 *}$, XIAOPING ZHAO ${ }^{2 *}$, YUHONG ZHOU ${ }^{3}$ and $\mathrm{YU} \mathrm{HU}^{1}$ \\ ${ }^{1}$ Department of Geriatrics, Zhongshan Hospital, Fudan University, Shanghai 200032; ${ }^{2}$ Department of Biochemistry \\ and Molecular Biology, Ninth People's Hospital, Shanghai JiaoTong University School of Medicine, Shanghai \\ 200011; ${ }^{3}$ Department of Oncology, Zhongshan Hospital, Fudan University, Shanghai 200032, P.R. China
}

Received February 21, 2012; Accepted May 10, 2012

DOI: $10.3892 / o r .2012 .1958$

\begin{abstract}
Colorectal cancer represents one of the most challenging diseases. Increasing evidence indicates that aberrant expression of microRNAs (miRNAs) is related to pathogenesis of colorectal cancer. Cancer cells reprogram metabolic pathways to sustain higher proliferation rates. Whether mechanisms underlying the role of miRNA in colorectal cancer are involved in metabolic reprogramming and the mechanisms through which miRNAs alter cancer metabolism are as yet unknown. Herein, we show that miR-124, miR-137 and miR-340 are associated with poor prognosis of colorectal cancer. Expression of these miRNAs inhibits the growth of colorectal cancer cells. PKM (pyruvate kinase isozyme) alternative splicing proteins (PTB1/ hnRNAPA1/hnRNAPA2), which control the inclusion of exon 9 (PKM1) or exon 10 (PKM2), are targeted by miR-124, miR-137 and miR-340. Consequently, miR-124, miR-137 and miR-340 switch PKM gene expression from PKM2 to PKM1. High ratios of PKM1/PKM2 inhibit the glycolysis rate, but elevate the glucose flux into oxidative phosphorylation. These results demonstrate that miRNAs (miR-124, miR-137 and miR-340) impair colorectal cancer growth by counteracting the Warburg effect due to regulating alternative splicing of the PKM gene.
\end{abstract}

\section{Introduction}

Colorectal cancer (CRC) is the third most common type of cancer. About 608,700 deaths from CRC occurred in 2008,

Correspondence to: Professor Yu Hu, Department of Geriatrics, Zhongshan Hospital, Fudan University, 180 Fenglin Road, Shanghai 200032, P.R. China

E-mail: hu.yu@zs-hospital.sh.cn

*Contributed equally

Abbreviations: CRC, colorectal cancer; miRNA, microRNA; PK, pyruvate kinase; hnRNP, heterogeneous nuclear ribonucleoprotein; PTB, polypyrimidine tract binding protein

Key words: miRNA, Warburg effect, pyruvate kinase isozyme, glycolysis, colorectal cancer accounting for $8 \%$ of all cancer deaths (1). About $30 \%$ of recurrent CRC patients have liver metastasis, and $>70 \%$ of them are not candidates for curative resection (2). In the past few years, accumulating evidence has suggested that microRNAs (miRNAs) are involved in the pathogenesis of CRC (3). The pattern of miRNA expression is related with cancer type, stage and other clinical variables, which makes miRNA a promising prognostic and therapeutic tool $(4,5)$.

In order to support high rates of proliferation, cancer cells consume additional nutrients and divert those nutrients into macromolecular synthesis pathways. Cancer cells prefer to metabolize glucose by glycolysis, intentionally avoid oxidative phosphorylation even when oxygen is abundant - the Warburg effect (6). Therefore, many cancer cells are characterized with increased lactate production and decreased $\mathrm{O}_{2}$ consumption (7). Active glycolysis coupled with increased glucose intake will provide sufficient intermediate metabolites, such as NADPH, acetyl-CoA and ribose, for biosynthetic process $(8,9)$.

Pyruvate kinase (PK), which converts phosphoenolpyruvate into pyruvate, is one of rate-limiting enzymes in glycolytic pathway.PKM is alternatively spliced to either M1 (PKM1) or M2 (PKM2) isoform, which contains exon 9 or exon 10, respectively $(10,11)$. The single exon difference endows the enzymes with distinct expression patterns and functions. PKM2 is exclusively expressed in embryonic, proliferating and cancer cells, which promotes glycolysis even in an aerobic environment. PKM1 is expressed in normal differentiated tissues, which promotes oxidative phosphorylation $(12,13)$. The expression PKM2 is critical for cancer cell growth. Switching PKM expression from PKM2 to PKM1 leads to inhibition of the Warburg effect and cancer growth (14).

Three heterogeneous nuclear ribonucleoproteins (hnRNPs) proteins, including polypyrimidine tract binding protein (PTB, also known as hnRNPI), hnRNPA1 and hnRNPA2, bind repressively to sequences flanking exon 9 . In the presence of PKM alternative splicing proteins (PTB1/hnRNAPA1/ hnRNAPA2), exon 10 is included in PKM transcript. To ensure high ratio of PKM2/PKM1 in cancer cells, expression of PTB1, hnRNAPA 1 and hnRNAPA 2 are tightly controlled by oncogenes, such as c-Myc. Importantly, the alternative splicing of PKM gene is important to sustain cancer cell growth through regulating glucose metabolism (15). 
In this study, we found that miR-124, miR-137 and miR-340 were associated with survival of CRC. Restoring the miRNA expression significantly inhibited growth of colorectal cancer cells. PKM alternative splicing proteins (PTB1/hnRNAPA1/ hnRNAPA2) were common targets of these miRNAs. miR-124, miR-137 and miR-340 promoted the alternative splicing of PKM gene to shift from PKM2 to PKM1. Therefore glucose was mainly metabolized through oxidative phosphorylation rather than glycolysis in the presence of miR-124, miR-137 and miR-340.

\section{Materials and methods}

Cell lines, reagents and tissue specimens. The colorectal cell lines HCT116, DLD1, SW480 and HT29, were purchased from ATCC. Cells were cultured in a humidified atmosphere of 95\% air, 5\% $\mathrm{CO}_{2}$, using recommended medium and 10\% FBS. Formalin-fixed paraffin-embedded (FFPE) colorectal cancer samples were collected from Zhongshan Hospital, Shanghai, China. The study protocol was reviewed and approved by the ethics committee of Zhongshan Hospital. miRIDIAN shMIMIC lentiviral microRNAs (OpenBiosystems) were used to express miRNAs in colorectal cancer cells. For miRNA activity assay, pmirGLO Dual-Luciferase miRNA target expression vector (Promega) was used.

miRNA profiling. Small RNA was extracted from FFPE tumor samples using High Pure miRNA isolation kit (Roche). RNA quality was assessed by absorbance spectrometry and electrophoresis on NanoDrop and Bioanalyzer 2100 instruments (Agilent). Agilent human genome microRNA microarray was used for miRNA profiling. Raw mean signal, total probe intensities and total gene intensities were uploaded into GeneSpring GX 10.0 software. Differences were considered statistically significant when the Benjamini-Hochberg false discovery ratecorrected $\mathrm{p}<0.05$.

Real-time RT-PCR. For microRNA, we used stem-loop quantitative RT-PCR. Briefly, 25 ng of total RNA was reverse transcribed using the miRCURY First-strand cDNA kit and the miRCURY microRNA primer sets. QPCR was performed with the Sequence Detection System 7900HT (Applied Biosystems) using the miRCURY LNA ${ }^{\mathrm{TM}}$ SYBR $^{\circledR}$ Green Master mix. The comparative $\mathrm{Ct}(\Delta \Delta \mathrm{Ct})$ method was used to determine the expression level of miRNA, and 5S RNA and U6B as endogenous controls (16-18).

Metabolism assay. Glycolysis rates were measured by following the conversion of $5-{ }^{3} \mathrm{H}$-glucose to ${ }^{3} \mathrm{H}_{2} \mathrm{O}$ as described previously (19). Briefly, cells were washed once in PBS before incubation in Krebs buffer without glucose for $30 \mathrm{~min}$ at $37^{\circ} \mathrm{C}$. The Krebs buffer was then replaced with Krebs buffer containing $10 \mathrm{mM}$ glucose spiked with $10 \mu \mathrm{Ci}$ of $5-{ }^{3} \mathrm{H}$-glucose. After $1 \mathrm{~h}$, triplicate samples of media were transferred to PCR tubes containing $0.2 \mathrm{~N} \mathrm{HCl}$ and the amount of ${ }^{3} \mathrm{H}_{2} \mathrm{O}$ generated which was determined by diffusion. Lactate was measured with a colorimetric kit according to the manufacturer's instructions (Biovision). Lactate production was normalized to the number of cells. Cellular oxygen consumption rates were measured using Biological oxygen consumption analysis system (Teket).
Statistical analysis. All statistical analysis was carried out using SPSS version 18.0 statistical software (SPSS). Two-tailed test and p-values $<0.05$ for significance were used.

\section{Results}

miRNA profiling in stage III colorectal cancer patients. Survival in patients with stage III colorectal cancer is totally different after standard treatment. Therefore, this cohort is quite suitable for prognostic analysis. miRNA expression profiles were evaluated in 12 subjects with stage III colorectal cancer. Thirty-two miRNAs showed $>1.5$-fold $(\mathrm{p}<0.05)$ difference. Compared with patients with $<5$ years survival, 21 miRNAs were up-regulated and 11 miRNAs were downregulated in patients with $\geq 5$-year survival. Expression map through unsupervised hierarchical clustering showed a clear separation of the two groups (Fig. 1A). To pursue underlying mechanisms, miRNA targets were analyzed by multiple databases (miRanda, TargetScanHuman and PicTar). Of 21 protective miRNAs, miR-124, miR-137 and miR-340 targeted the alternative splicing proteins of PKM: PTB1/hnRNAPA1/ hnRNAPA2. This group of proteins has recently been found to regulate the Warburg effect, which is critical for cancer growth (20). To better understand the role of miRNA in cancer metabolism, miR-124, miR-137 and miR-340 were chosen for further study.

Growth inhibition of colorectal cancer cells by miR-124, $m i R-137$ and miR-340. Poor prognosis is related with multiple aspects of colorectal cancer, including proliferation, survival and invasion. miRNA target prediction suggested that miR-124, miR-137 and miR-340 were probably involved in cancer metabolic reprogramming. The main aim of metabolic reprogramming is to sustain rapid cancer growth. Then we asked whether growth of colorectal cancer cells was regulated by these miRNAs. HCT116 cells were infected with lentivirus containing the precursor of miRNA or non-silencing control sequence (NS) (Fig. 1B). MiR-145, as a positive control, was known to have potent antiproliferative activity in colorectal cancer (21-23). miR-124, miR-137 or miR-145 inhibited the growth of HCT116 cells. Growth inhibition was more significant in cells treated with three miRNAs simultaneously (Fig. 1C). Consistent results were obtained in other colorectal cancer cell lines (Fig. 1E). To further corroborate the antiproliferative role of miR-124, miR-137 and miR-340 we used the miRNA specific inhibitor LNA (locked nucleic acid), to reverse miRNA-mediated growth inhibition. After 2 weeks of puromycin selection, miRNA stably expressed HCT116 cells were obtained. Then these cells were transfected with specific LNAs, respectively. LNA rescued cells from miRNA-induced growth inhibition (Fig. 1D). Therefore, miR-124, miR-137 and miR-340 had important roles of regulating colorectal cancer cell growth.

PTB1, hnRNAPA1 and hnRNAPA2 are targeted by miR-124, $m i R-137$ and $m i R-340$. miRNA target prediction indicated that PTB1, hnRNAPA1 and hnRNAPA2 contained binding sites of these miRNAs. The protein level of PTB1 was significantly reduced in HCT116 cells transfected by miR-124, miR-137 or miR-340. miR-124 or miR-340 repressed expression of 
A

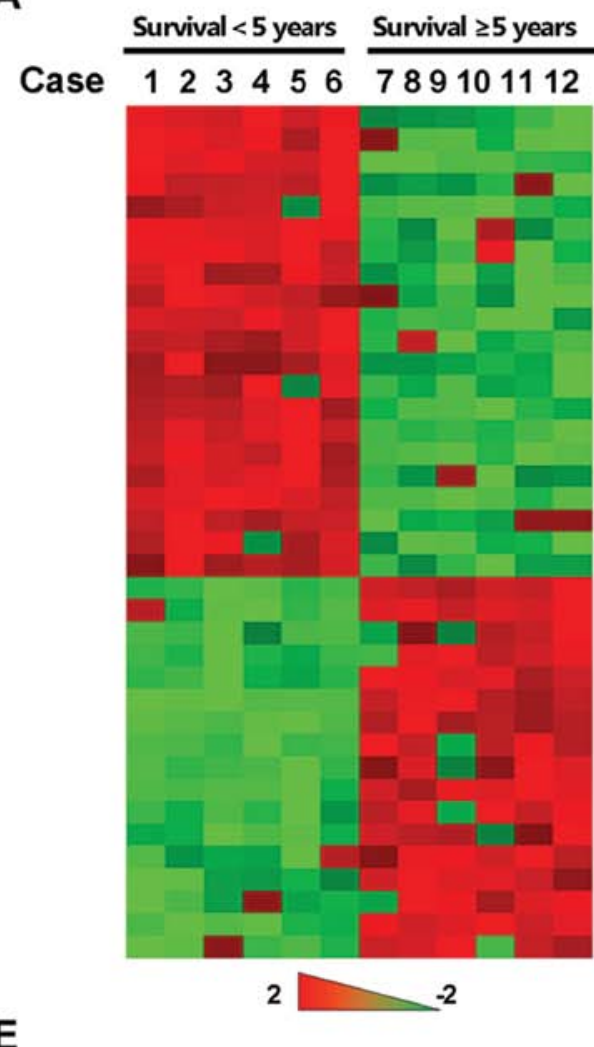

DLD1

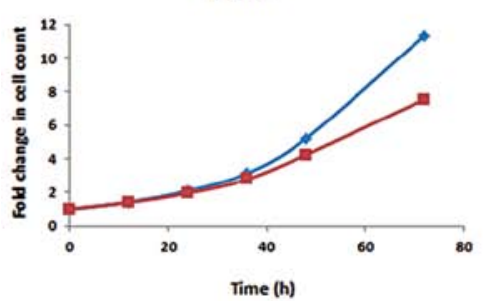

\section{Cluster analysis}

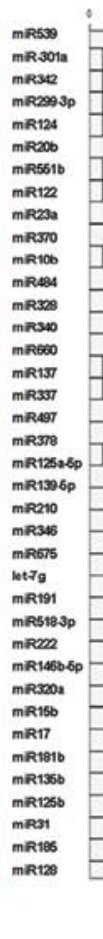

B

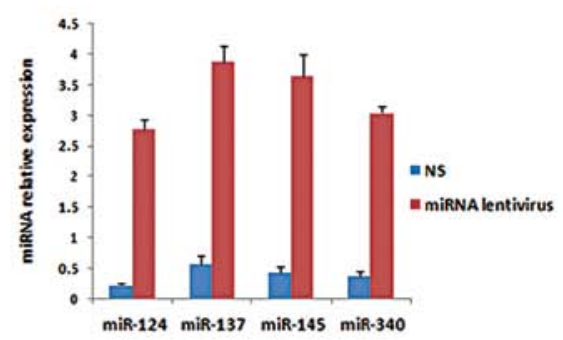

C

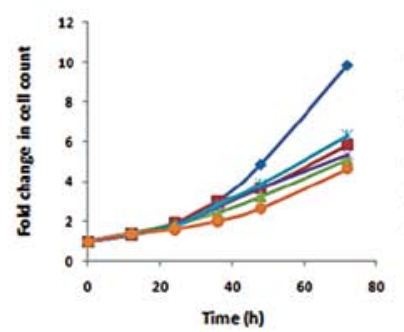

$\rightarrow$ NS

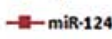

$\rightarrow-\operatorname{miR} \cdot 137$

-mir.145

\#-mir.340 $\rightarrow-\operatorname{miR} \cdot 124 / 137 / 340$

D

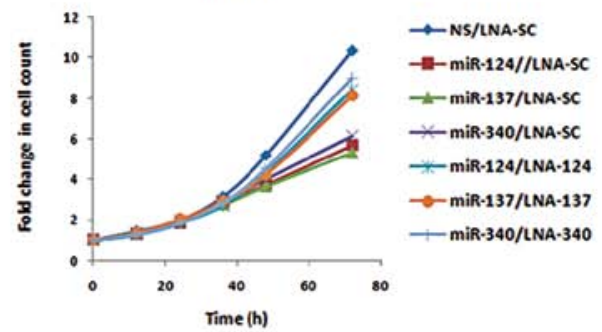

HT29
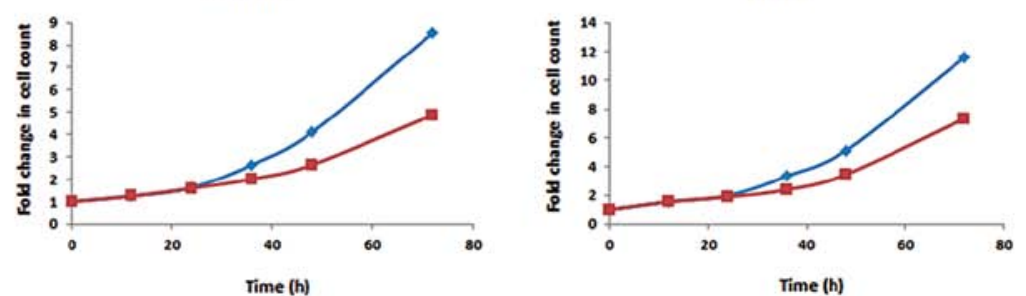

$\rightarrow$ NS

$\rightarrow-\operatorname{miR} \cdot 124 / 137 / 340$

Figure 1. miR-124, miR-137 and miR-340 inhibited proliferation of colorectal cancer cells. (A) Heatmap and hierarchical clustering of significantly differentially expressed miRNAs, patients in columns and miRNA in rows. miRNA expression data are shown relative to the average value of normal colorectal tissue. The expression values ranged from $+2 \log _{10}$ to $-2 \log _{10}$. (B) HCT116 cells were infected by lentivirus with miR-124, miR-137, miR-145 or miR-340, respectively, NS as a control. miRNA levels were assessed by QPCR, 5s rRNA, 18s rRNA and U6 as global median-normalization references. (C) HCT116 cells were treated by lentivirus with indicated miRNA or NS for proliferation assay. Cell counts were performed every $12 \mathrm{~h}$ for 3-5 days. Time zero was defined as $12 \mathrm{~h}$ after seeding. Proliferation curves were drawn according to the fold of cell counts compared with time zero point. (D) miRNA stable expressing HCT116 cells were treated with LNA oligonucleotide complementary to the specific miRNA (LNA-124, LNA -370 or LNA -137) or control LNA oligonucleotide (LNA-SC). Cell proliferation was then analyzed by cell counting. (E) Cell proliferation was analyzed in DLD1, SW480 and HT29 cells transfected with miR-124/137/340 or NS.

hnRNAP2 protein, and hnRNAP1 was targeted by miR-137 (Fig. 2A). Consistent results were also found in mRNA level of target genes (Fig. 2B). To verify these transcripts targeted by miRNAs, 3'UTR luciferase reporter plasmids were constructed. Mutation sites were selected according to their evolutionary conservation. Constructs with intact miRNA binding sites of PTB1 were expressed at significantly lower levels of luciferase activity in the presence of these miRNAs. miR-124 and miR-340 repressed luciferase activity of wild type reporter of hnRNAP2. Only miR-137 repressed luciferase activity of wild-type reporter of hnRNAP1 (Fig. 2C). Moreover, expression of PTB1, hnRNAP1 or hnRNAP2 compromised the growth inhibition induced by these miRNAs (Fig. 2D). These data indicated that the PKM alternative splicing proteins were required for miRNA-mediated growth inhibition of colorectal cancer cells.
miR-124, miR-137 and miR-340 regulate PKM alternative splicing. The two isoforms, PKM1 and PKM2, were produced from mutually exclusive alternative splicing of PKM gene. This process is mainly regulated by PTB1, hnRNAPA1 and hnRNAPA2 (15). Cancer cells were characterized by switching PKM1 to PKM2 expression. PKM1 or PKM2 was assayed by specific exon-overlapping primers, respectively. miR-124, miR-137 and miR-340 induced an increase of PKM1 mRNA, and concurrent decrease of PKM2 mRNA (Fig. 3A). The ratio of PKM1/PKM2 was significantly increased in cells treated by three miRNAs simultaneously (Fig. 3B). Consistent results were obtained by analyzing their protein levels (Fig. 3C). Next we asked whether the switch from PKM2 to PKM1 was essential for miRNA-mediated growth regulation. Knockdown of PKM2 by siRNA in HCT116 cells resulted in growth inhibition, which was consistent with published reports $(10,14)$. In the absence of 
A

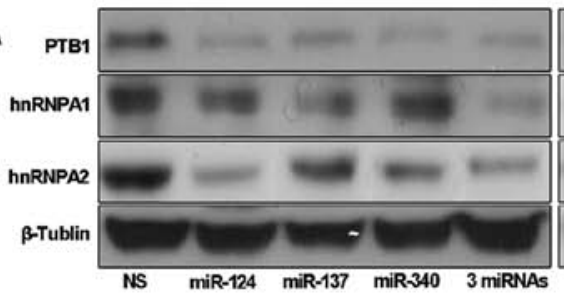

B

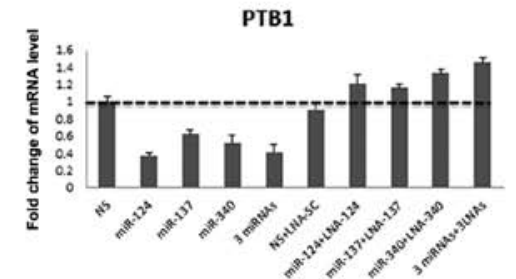

C

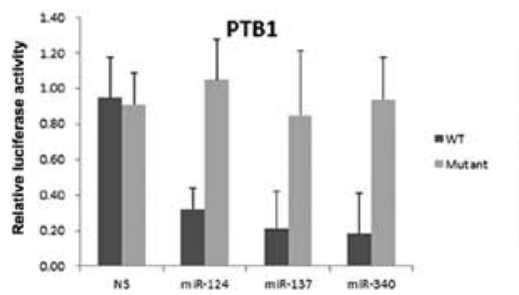

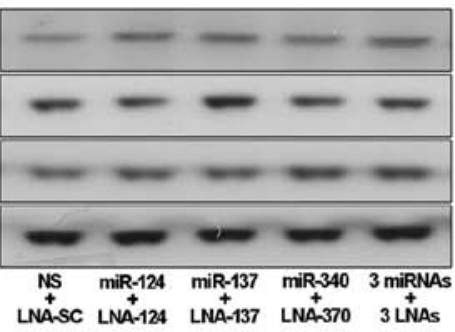

hnRNPA1
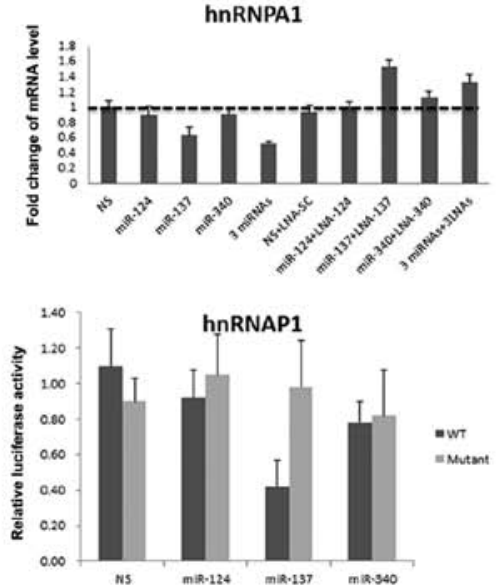

D
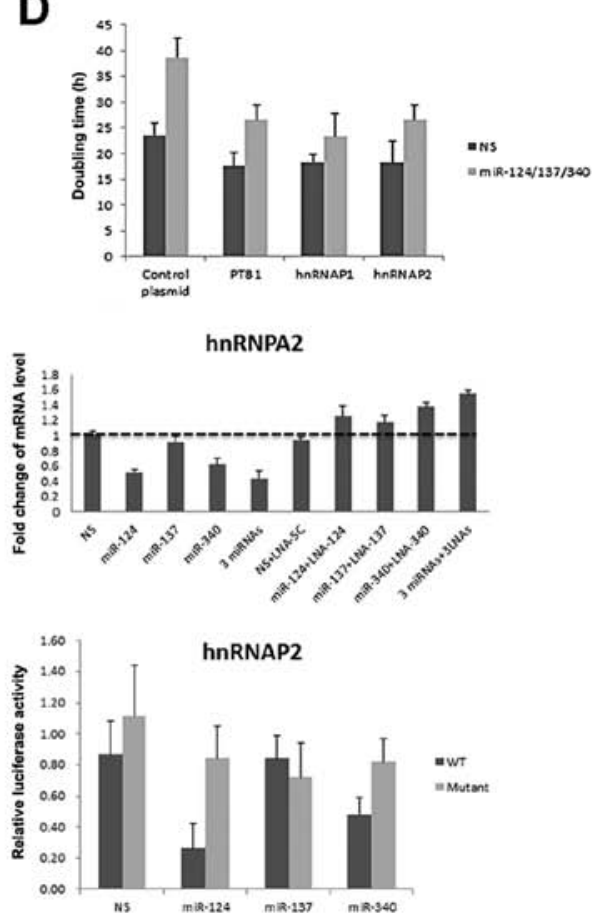

Figure 2. PTB1, hnRNAPA1 and hnRNAPA2 are targets of miR-124, miR-137 and miR-340. (A) Immunoblotting of miRNA stably expressing HCT116 cells with PTB1, hnRNAPA1, hnRNAPA2 and $\beta$-tublin antibodies. (B) mRNA levels of PTB1, hnRNAP1 or hnRNAP2 were analyzed in cells with indicated treatment. Expression value was calculated with the $\triangle \Delta C T$ method. (C) Relative firefly luciferase activity derived from PTB1, hnRNAP1, hnRNAP2 3'UTR wild-type or mutant reporter constructs was analyzed in miRNA stably expressing HCT116 cells. All values were normalized to renilla luciferase activity. (D) HCT116 cells were treated with mixture of miR-124/137/340 or NS control for $24 \mathrm{~h}$, then transfected with PTB1, hnRNAP1, hnRNAP2 or control plasmid, respectively. Doubling time was calculated by cell counting. Error bars represent standard deviations from 3 independent experiments. 3-miRNAs indicate combination of miR-124, miR-137 and miR-340. 3-LNAs indicate combination of three LNA miRNA inhibitors.
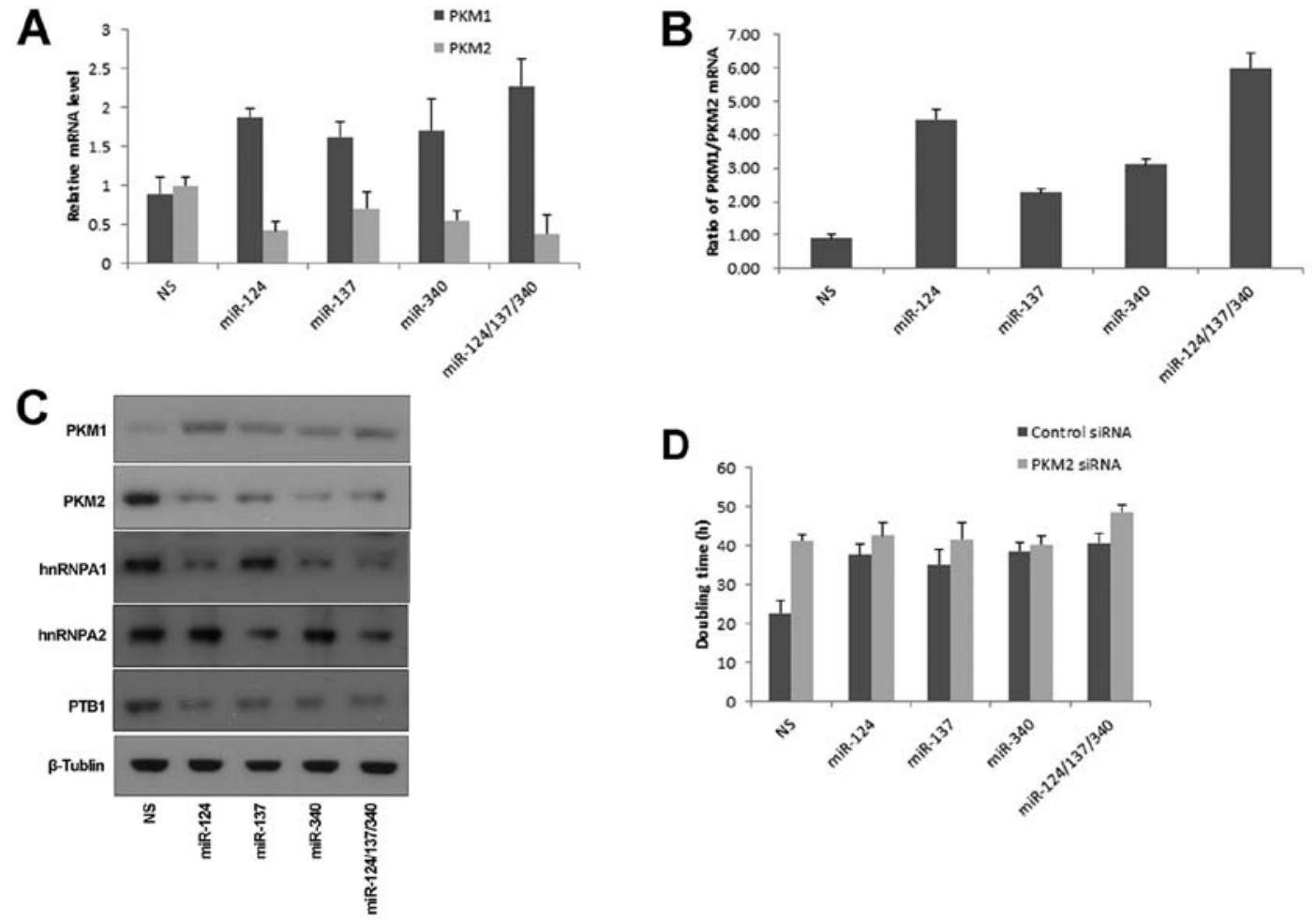

Figure 3. miR-124, miR-137 and miR-340 induce the switch of PKM expression to PKM1. (A) mRNA levels of PKM1 and PKM2 were analyzed by QPCR in cells transfected with NS, miR-124, miR-137, miR-340 or mixture of these three miRNAs. Relative mRNA level was obtained by the $\Delta \Delta C T$ method, normalized by global control ( $\beta$-tublin, actin and 18s rRNA). NS treated cells as a reference. (B) Ratio of PKM1/PKM2 was calculated by their relative mRNA levels. (C) Immunoblotting assessed PKM1 and PKM2 protein expression in HCT116 cells transfected by the indicated miRNA. (D) HCT116 cells were transfected with PKM2 siRNA or control siRNA for $24 \mathrm{~h}$. Then cells were treated with miRNA or control lentivirus. Doubling time was calculated by cell counting. Error bars represent standard deviations from 3 independent experiments. 

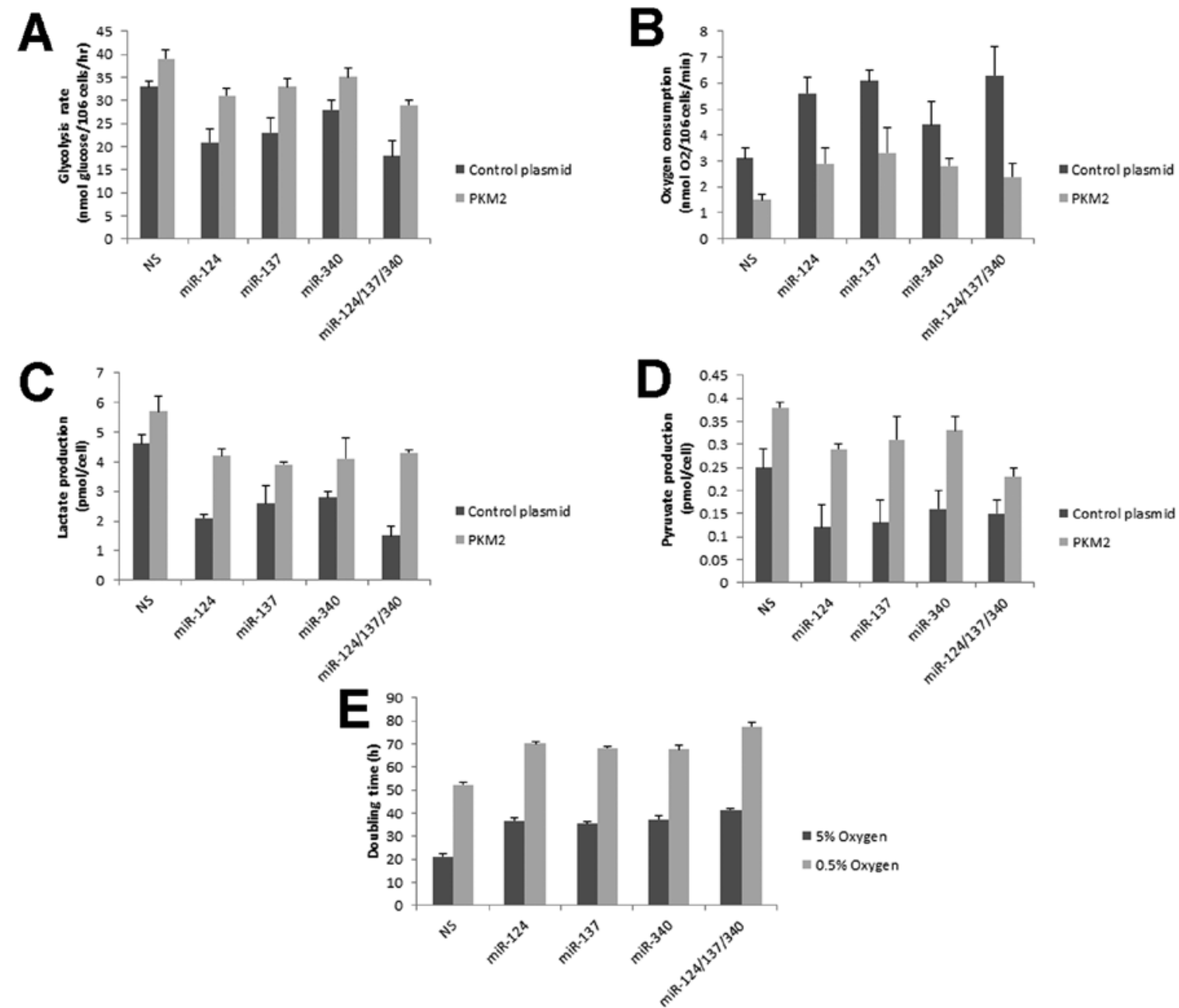

Figure 4. miR-124, miR-137 and miR-340 inhibit aerobic glycolysis in HCT116 cells. (A) Glycolytic rates of HCT116 cells expressing indicated miRNA with or without PKM2 were measured by ${ }^{3} \mathrm{H}_{2} \mathrm{O}$ production from $5-{ }^{3} \mathrm{H}$-glucose. (B) Oxygen consumption was analyzed in HCT116 cells. (C and D) Lactate and pyruvate production was analyzed by colorimetric method, and normalized to cell number. (E) Cell growth was analyzed by doubling time in HCT116 cells with 5\% or $0.5 \%$ oxygen condition. Error bars represent standard deviations from 3 independent experiments.

PKM2, these miRNAs had no effect on cell growth (Fig. 3D). These data indicated that miR-124, miR-137 and miR-340 inhibited colorectal cancer growth through regulating PKM alternative splicing.

Aerobic glycolysis is inhibited by miR-124, miR-137 and $m i R-340$. PKM2, as a key enzyme for glycolysis, is selectively expressed in cancer cells and is critical for cell proliferation $(9,24)$. Cellular glycolytic rate was measured by conversion of $5-{ }^{3} \mathrm{H}$-glucose to ${ }^{3} \mathrm{H}_{2} \mathrm{O}(14,25,26)$. miR-124, miR-137 and miR-340 showed a decrease of the glycolytic rate (Fig. 4A). Switching to PKM1 expression resulted in cells preferentially metabolizing glucose by oxidative phosphorylation rather than glycolysis. Oxygen consumption was increased in the presence of miR-124, miR-137 and miR-340 (Fig. 4B). Consistently, these miRNAs promoted cells to convert less pyruvate to lactate (Fig. 4C). Pyruvate was also decreased in these miRNA-treated cells (Fig. 4D) due to higher activity of PKM1 than PKM2 (12). On the other hand, recovering PKM2 expression compromised the miRNA-mediated glycolytic reprogramming (Fig. 4A-D). In lower oxygen condition, energy generation is strongly dependent on glycolysis. Compared with normoxia, miRNAs induced more apparent growth inhibition in hypoxia (Fig. 4E). Taken together, these data suggested that glucose metabolism of colorectal cells were regulated by miR-124, miR-137 and miR-340.

\section{Discussion}

Currently, it is still hard to predict the outcome of colorectal cancer depending on traditional pathological variables, including tumor size, lymph node status and tumor grade. Survival rates are totally different among stage III patients. After curative surgery, stage III patients experience 50-60\% chance of recurrence (27). Therefore, analysis of genetic and epigenetic changes of stage III CRC was very informative. In the present study, miRNA expression profiling of stage III $\mathrm{CRC}$ was performed using a microarray approach. Aberrantly expressed miRNAs associated with survival were identified. Twenty-one miRNAs, as anti-oncomirs, were decreased $>1.5$-fold in $\geq 5$ years in CRC patients. Using miRNA target predication databases, 3 anti-oncomirs (miR-124, miR-137 and miR-340) were shown to target the PKM alternative splicing proteins (PTB1/hnRNAPA1/hnRNAPA2). 


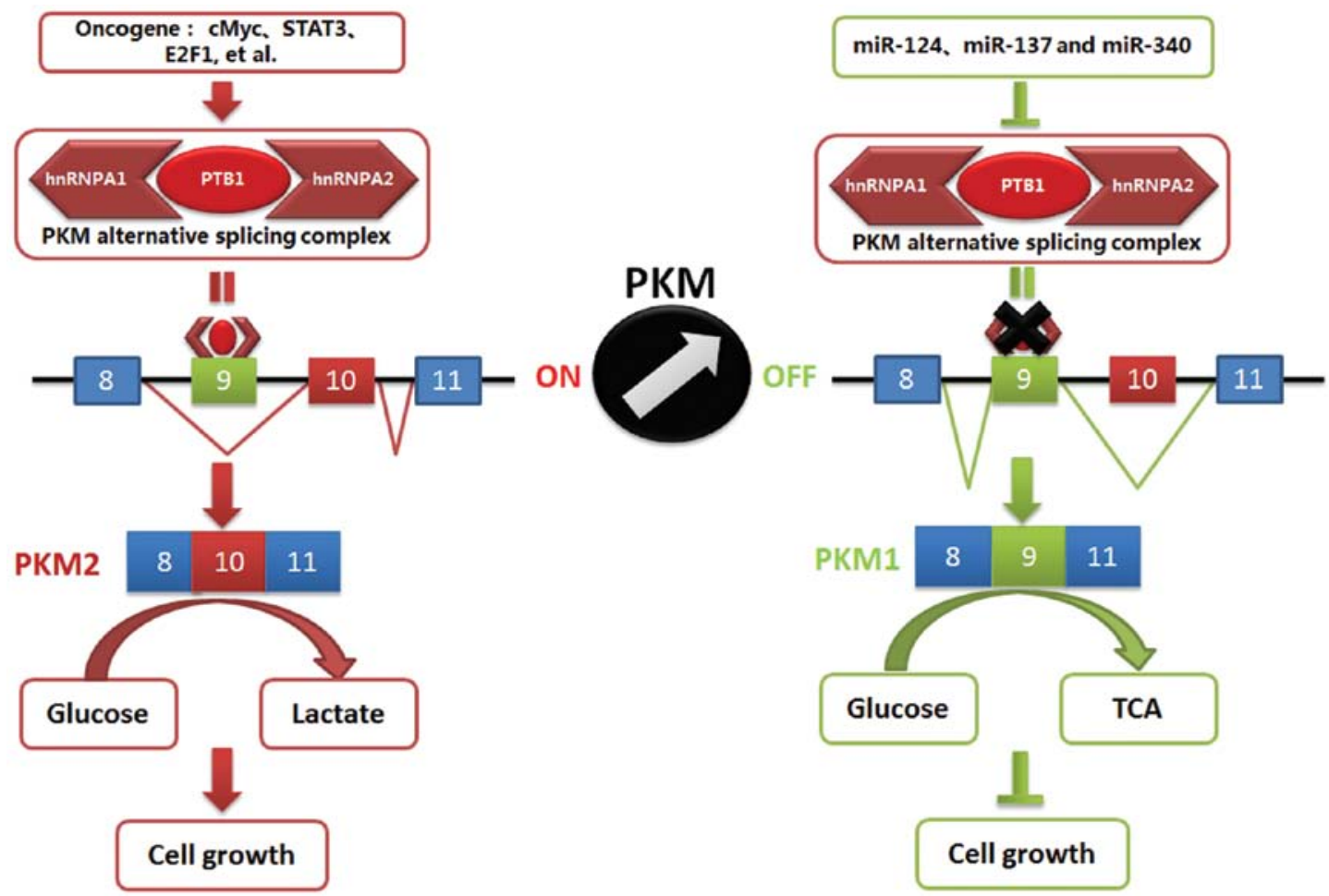

Figure 5. A model of miR-124, miR-137 and miR-340 regulating CRC cell growth.

Luciferase reporter and protein level analysis demonstrated that miR-124, miR-137 and miR-340 regulated the expression of PKM alternative splicing proteins. PTB1 was a target of miR-124, miR-137 and miR-340. Also, miR-124 and miR-340 directly regulated expression of hnRNAP2. HnRNAP1 was only targeted by miR-137. miRNA targets were further validated by mutating conserved binding sites for miRNA. PTB1 expression is under control of miR-124, which is important for development of the nervous system (28). In the same manner, miR-124 regulates amyloid precursor protein expression via targeting PTB1-mediated alternative splicing (29). Trans- $\beta$-nitrostyrene (TBNS), as a potent inhibitor of protein phosphatase PTB1, displays an antiproliferative effect in colorectal cancer (30). Our data indicated that expression of PTB1, hnRNAPA1 or hnRNAPA 2 abolished the miRNAinduced growth inhibition of colorectal cancer. Besides miRNA regulation, the expression of PKM alternative splicing proteins are also regulated by the upstream oncogene c-Myc (15). Therefore, c-Myc could regulate cancer growth directly through PKM alternative splicing proteins or indirectly through miR-124, miR-137 and miR-340.

Alternative splicing of PKM is involved in determining the metabolic phenotype of mammalian cells. PKM2 expression in cancer cells has an important role in maintaining sufficient materials for rapid proliferation. Here we found that miR-124, miR-137 and miR-340 switched PKM expression from PKM2 to PKM1 isoform. Ratio of PKM1/PKM2 was more dramatically increased with miR-124/137/340 co-transfection, compared to single miRNA treatment. Growth regulation induced by these miRNAs was dependent on PKM2 expression. Knockdown of PKM2 compromised miRNA-induced growth inhibition. For cancer cells, PKM2 not only supports cell growth via Warburg effect as a metabolic enzyme, but also promotes transactiva- tion of HIF-1 target genes as a transcription coactivator (31). It implied that other mechanisms were probably also involved. PKM2, as a transcription cofactor, might regulate miR-124, miR-137 and miR-340 expression in the transcription level.

Warburg and colleagues discovered that many tumors prefer glycolysis, even in normal oxygen level. Higher glycolysis and glucose consumption support energy for proliferation and provide catabolic intermediate to fuel lipid and nucleic acid biosynthesis. Recently, several groups have found that the mechanism of the Warburg effect is via PKM alternative splicing mechanism $(14,15,31)$. Several indicators of glycolysis, including glycolytic rate, oxygen consumption and intermediate metabolites, showed that Warburg effect was inhibited by miR-124, miR-137 and miR-340. These miRNAs diverted the glucose into oxidative phosphorylation rather than glycolysis. In this case, short or intermediate metabolites for anabolic processes impeded the colorectal cancer cell growth. Of note, miRNA-mediated growth inhibition was more apparent under hypoxic condition compared with normoxic condition.

As shown in Fig. 5, miR-124, miR-137 and miR-340 played important roles in regulating colorectal cancer growth. The underlying mechanism was involved in repressing the Warburg effect through switching PKM expression.

\section{Acknowledgements}

This work was supported by the National Natural Science Foundation of China grant (no. 81001008).

\section{References}

1. American Cancer Society: NY: Global Cancer Facts \& Figures, 2nd edition. http://www.cancer.org/Research/CancerFactsFigures/ GlobalCancerFactsFigures/index. Accessed 2008. 
2. Malafosse R, Penna C, Sa Cunha A and Nordlinger B: Surgical management of hepatic metastases from colorectal malignancies. Ann Oncol 12: 887-894, 2001

3. Lu J, Getz G, Miska EA, et al: MicroRNA expression profiles classify human cancers. Nature 435: 834-838, 2005.

4. Lee YS and Dutta A: MicroRNAs in cancer. Annu Rev Pathol 4: 199-227, 2009.

5. Schetter AJ, Leung SY, Sohn JJ, et al: MicroRNA expression profiles associated with prognosis and therapeutic outcome in colon adenocarcinoma. JAMA 299: 425-436, 2008.

6. Warburg O: On the origin of cancer cells. Science 123: 309-314, 1956.

7. Gatenby RA and Gillies RJ: Why do cancers have high aerobic glycolysis? Nat Rev Cancer 4: 891-899, 2004.

8. Jones RG and Thompson CB: Tumor suppressors and cell metabolism: a recipe for cancer growth. Gene Dev 23: 537-548, 2009.

9. Vander Heiden MG, Cantley LC and Thompson CB: Understanding the Warburg effect: the metabolic requirements of cell proliferation. Science 324: 1029-1033, 2009.

10. Mazurek S, Boschek CB, Hugo F and Eigenbrodt E: Pyruvate kinase type $\mathrm{M} 2$ and its role in tumor growth and spreading. Semin Cancer Biol 15: 300-308, 2005.

11. Noguchi T, Inoue $\mathrm{H}$ and Tanaka T: The M1- and M2-type isozymes of rat pyruvate kinase are produced from the same gene by alternative RNA splicing. J Biol Chem 261: 13807-13812, 1986.

12. Christofk HR, Vander Heiden MG, Wu N, Asara JM and Cantley LC: Pyruvate kinase M2 is a phosphotyrosine-binding protein. Nature 452: 181-186, 2008.

13. Vander Heiden MG, Locasale JW, Swanson KD, et al: Evidence for an alternative glycolytic pathway in rapidly proliferating cells. Science 329: 1492-1499, 2010.

14. Christofk HR, Vander Heiden MG, Harris MH, et al: The M2 splice isoform of pyruvate kinase is important for cancer metabolism and tumour growth. Nature 452: 230-233, 2008.

15. David CJ, Chen M, Assanah M, Canoll P and Manley JL: HnRNP proteins controlled by c-Myc deregulate pyruvate kinase mRNA splicing in cancer. Nature 463: 364-368, 2010.

16. Wang BD, Kline CL, Pastor DM, et al: Prostate apoptosis response protein 4 sensitizes human colon cancer cells to chemotherapeutic $5-\mathrm{FU}$ through mediation of an NF kappaB and microRNA network. Mol Cancer 9: 98, 2010.

17. Patnaik SK, Kannisto E, Knudsen S and Yendamuri S: Evaluation of microRNA expression profiles that may predict recurrence of localized stage I non-small cell lung cancer after surgical resection. Cancer Res 70: 36-45, 2010.
18. Silber J, Lim DA, Petritsch C, et al: miR-124 and miR-137 inhibit proliferation of glioblastoma multiforme cells and induce differentiation of brain tumor stem cells. BMC Med 6: 14, 2008.

19. Vanderweele DJ and Rudin CM: Mammalian target of rapamycin promotes vincristine resistance through multiple mechanisms independent of maintained glycolytic rate. Mol Cancer Res 3: 635-644, 2005.

20. Chen M, Zhang J and Manley JL: Turning on a fuel switch of cancer: hnRNP proteins regulate alternative splicing of pyruvate kinase mRNA. Cancer Res 70: 8977-8980, 2010.

21. Zhang J, Guo H, Qian G, et al: MiR-145, a new regulator of the DNA fragmentation factor-45 (DFF45)-mediated apoptotic network. Mol Cancer 9: 211, 2010

22. Akao Y, Nakagawa Y, Hirata I, et al: Role of anti-oncomirs miR-143 and -145 in human colorectal tumors. Cancer Gene Ther 17: 398-408, 2010.

23. Gregersen LH, Jacobsen AB, Frankel LB, Wen J, Krogh A and Lund AH: MicroRNA-145 targets YES and STAT1 in colon cancer cells. PLoS One 5: e8836, 2010.

24. Kaelin WG Jr and Thompson CB: Q\&A: Cancer: clues from cell metabolism. Nature 465: 562-564, 2010.

25. Vander Heiden MG, Plas DR, Rathmell JC, Fox CJ, Harris MH and Thompson CB: Growth factors can influence cell growth and survival through effects on glucose metabolism. Mol Cell Biol 21: 5899-5912, 2001.

26. Ashcroft SJ, Weerasinghe LC, Bassett JM and Randle PJ: The pentose cycle and insulin release in mouse pancreatic islets. Biochem J 126: 525-532, 1972.

27. Center MM, Jemal A, Smith RA and Ward E: Worldwide variations in colorectal cancer. CA Cancer J Clin 59: 366-378, 2009.

28. Makeyev EV, Zhang J, Carrasco MA and Maniatis T: The MicroRNA miR-124 promotes neuronal differentiation by triggering brain-specific alternative pre-mRNA splicing. Mol Cell 27: 435-448, 2007.

29. Smith P, Al Hashimi A, Girard J, Delay C and Hebert SS: In vivo regulation of amyloid precursor protein neuronal splicing by microRNAs. J Neurochem 116: 240-247, 2011.

30. Werner JM, Eger K and Jurgen Steinfelder H: Comparison of the rapid pro-apoptotic effect of trans-beta-nitrostyrenes with delayed apoptosis induced by the standard agent 5-fluorouracil in colon cancer cells. Apoptosis 12: 235-246, 2007.

31. Luo $\mathrm{W}, \mathrm{Hu} \mathrm{H}$, Chang R, et al: Pyruvate kinase M2 is a PHD3stimulated coactivator for hypoxia-inducible factor 1. Cell 145: 732-744, 2011. 